\title{
SimBetAge: Utilizing Temporal Changes in Social Networks for Delay/Disconnection Tolerant Networking
}

\author{
Jó Ágila Bitsch Link, Nicolai Viol, André Goliath, Klaus Wehrle \\ Distributed Systems Group \\ RWTH Aachen University \\ Ahornstr. 55 \\ 52074 Aachen, Germany \\ (bitsch|viol|goliath|wehrle)@cs.rwth-aachen.de
}

\begin{abstract}
In this paper, we present SimBetAge, an extension to SimBet taking into account the gradual aging of connections in social networks which thereby increases the performance by an order of magnitude, especially in evolving network structures. For this purpose, we redefine similarity and betweenness to make use of weighted social network graphs.
\end{abstract}

\section{Categories and Subject Descriptors}

C.2.1 [Network Architecture and Design]: Store and forward networks

\section{General Terms}

Algorithms, Design, Performance

\section{INTRODUCTION}

Communication networks seem to be one of the defining technologies of our time, especially nowadays with the advent of wireless local area networking standards like IEEE 802.11 and short range personal area network standards like Bluetooth. Almost all new personal electronic devices are equipped with communication capabilities of some kind.

Nevertheless, access to infrastructure networks is not necessarily available in all situations where communication is desirable. In these situations mobile ad-hoc network protocols provide the means to communicate with devices reachable either directly or via (possibly a range of) directly reachable devices. While the Manet approach does not require infrastructure, it still depends on a fully connected end-to-end path.

The introduction of delay and disruption tolerant networks (DTN) removes this dependency by allowing the delivery of data over sporadical connected networks. Data bundles are cached for a possibly long time before a suitable contact to a forwarding node is made.

This work discusses SimBetAge, our heuristic approach to forwarding data based on the analysis of the social relations and social network underlying the contacts between nodes. Building on top of SimBet[1], we extend the representation of dynamics in social networks.

\section{CONCEPT}

The challenging part in a DTN multi-hop routing scheme is to assign the next hop. In general, the complexity of this problem is strongly related to the complexity of the network, i.e. uncertainties in connectivity and movement of nodes. If the dynamic and link instability of a network becomes too high, traditional routing strategies based on link-state schemes will fail due to the frequency of changes. To cope with these uncertainties we need to discover some structures that help to decide which neighbor is an appropriate next hop.

In [3], the authors presented a probabilistic routing scheme called PRoPHET where each node keeps a table with probabilities for reaching every possible destination in the network and making routing decisions depending on the frequency of observations, the time of absence and the distance to a destination.

In [1] Daly, et al. presented a novel routing approach called SimBet which takes common properties of social networks such as social similarity and betweenness centrality into account. The motivation on SimBet is to use social network analysis to provide an efficient routing scheme that has no need of global knowledge about the network as PRoPHET requires.

Conceptually, a node forwards messages to the node that has a higher social similarity to the destination, i.e. it is more likely to have a contact to the destination. If there is no such node known to the forwarder, it instead forwards messages to nodes with a higher betweenness, i.e. to a potentially better forwarder to other parts/clusters in the network.

Despite the fact that SimBet performs quite well as a result of the evaluations of the paper [1], some weaknesses can be pointed out. In SimBet, routing decisions are made upon comparing the social relations and roles of individual nodes. However, some relations are stronger than others, i.e. contacts between them are more frequent. Also, social relations are evolving over time or may change completely. Therefore, a simple statement that $x$ knows $y$ without additional qualifiers is not enough.

Also a node might have different roles: It may participate in different clusters (different circles of friends) so one node might be important for forwarding information from one cluster to another but not necessarily that important for forwarding within a cluster. Additionaly, if not only short periods need to be considered, SimBet suffers from not taking changes over time into account.

Bubble Rap [2] does take different roles into account by forming communities based on the nodes social relations. But again, once a snapshot of the social structure is taken these structures are not adapted. 


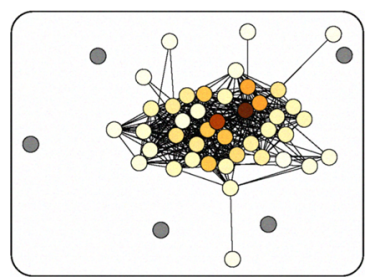

Figure 1: Social structure changing over time

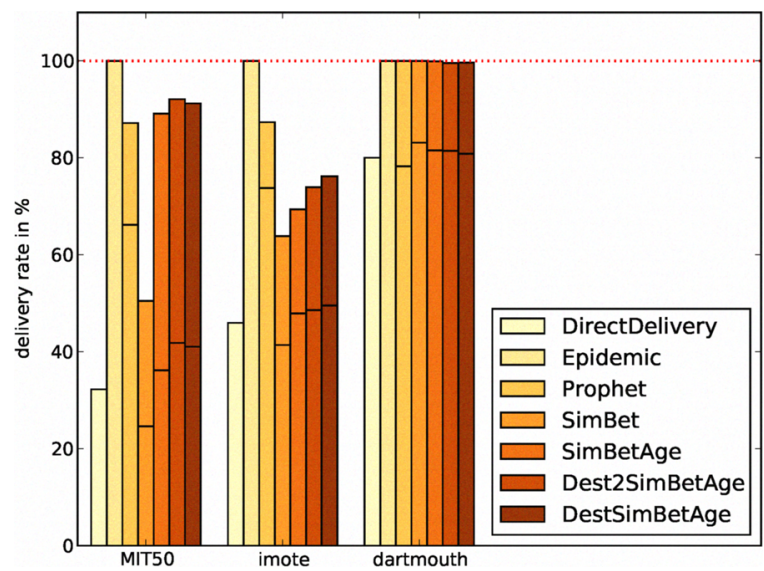

Figure 2: Delivery rates for different algorithms.

To levitate these shortcomings we present SimBetAge. Intuition demands for dynamics and degrees in the relational model: People might have been best friends in kindergarten but have not seen each other in years now. Data from longer running traces show that these variations indeed reflects in the network structure. With SimBetAge we present an approach to allow reacting to changes over time in the social structure.

\subsection{Modelling the dynamics in relations}

A binary model of a social relation, i.e. one node knows another node or not, does not cope with the dynamics a social network typical has.

Using a weighted graph $G(t)$ as a model of a social network will bring a more realistic view. Were $G(t)=(V, E, \omega(e, t))$ is a fully connected graph with $V$ vertices, $E$ edges and $\omega(e, t) \mapsto[0,1]$ is a mapping for every edge $e \in E$ and time $t \in T$ to a weight. This weight of an edge is what we will call the freshness of an edge, with 0 representing no and 1 being a permanent connection between two nodes (compare to $[3])$.
The freshness of a single contact is influenced by two events. Every time step event will decrease the freshness value while an encounter event will increase the freshness of that edge. Due to the representation by logistic growth and exponential decay for the freshness value of a single edge, the freshness value $\omega(e, t)$ can also be interpreted as an indicator, how probable it is that the two nodes $u$ and $v$ are connected at time $t$.

In a binary graph the similarity $S_{\text {binary }}(u, v)=\mid N(u) \cap$ $N(v) \mid$ of two nodes $u$ and $v$ is defined by the number of common neighbors between $u$ and $v$. In a graph with aged relations, we define the similarity of two nodes as being proportional to the freshness of their concurrent neighborhood. Therefore, we need to consider that the encounter information at node $u$ about node $v$ might be outdated. That means, the similarity of node $u$ to node $v$ concerning $w$ needs to be rated by the age of the encounter information of node $v$ about the common neighbor $w$.

As communication not only takes place via shortest paths, the network flow needs to be considered. This leads to the notion of flow betweenness, which we in turn use as centrality measure in lieu of betweenness centrality.

\section{RESULTS}

As shown in figure 1, which is based on data by the Haggle project [4], the ego betweenness values heavily change over time. Since our algorithm takes these changes into account we can react to them and make smarter routing decisions.

We performed a number of further simulations and evaluations which lead to the conclusion that taking the freshness of node relations into account leads to a significantly better performance than a binary representation (see figure 2). Further work shows that the directed betweenness (not discussed here) is another very useful addition to SimBetAge and increases performance significantly.

\section{REFERENCES}

[1] E. M. Daly and M. Haahr. Social network analysis for routing in disconnected delay-tolerant manets. In MobiHoc '07: Proc. of the 8th ACM international symposium on Mobile ad hoc networking and computing, pages 32-40, Montreal, Quebec, Canada, 2007.

[2] P. Hui, J. Crowcroft, and E. Yoneki. Bubble rap: social-based forwarding in delay tolerant networks. In MobiHoc '08: Proc. of the 9th ACM international symposium on Mobile ad hoc networking and computing, pages 241-250, New York, NY, USA, 2008.

[3] A. Lindgren, A. Doria, and O. Schelén. Probabilistic routing in intermittently connected networks. In Service Assurance with Partial and Intermittent Resources, volume 3126/2004 of Lecture Notes in Computer Science, pages 239-254. Springer Berlin / Heidelberg, 2004.

[4] J. Scott, R. Gass, J. Crowcroft, P. Hui, C. Diot, and A. Chaintreau. CRAWDAD data set cambridge/haggle (v. 2006-09-15). Downloaded from http://crawdad.cs.dartmouth.edu/cambridge/haggle, 2006.

\section{Webpage}

More information about this work and can be found on: http://www.ds-group.info/research/projects/ratpack/ 